\title{
Quarter Symmetric Connections On Complex Weyl Manifolds
}

\author{
İlhan Gül \\ İhan Gül: Department of Mathematics, Istanbul Technical University, Istanbul, e- \\ mail:igul@itu.edu.tr
}

Abstract. In this work, we study quarter symmetric linear connections on Kähler Weyl manifolds and almost contact Weyl manifolds.

Keywords. Weyl manifold · Kähler Weyl manifold · Almost contact Weyl manifold . Quarter symmetric linear connection.

MSC 2010 Classification. Primary: 53C15; Secondary:53B05.

\section{$1 \quad$ INTRODUCTION}

A gauge invariant theory which unifies the gravity and the electromagnetic fields was first introduced by Weyl in 1918 [13]. For physical reasons, his theory was not accepted but it remained both as a part of physics and mathematics. Weyl manifold is a differentiable manifold with a torsion free connection which is non-metric.

In 1924, Friedmann and Schouten introduced a semi-symmetric linear connection in a differentiable manifold [4]. After that, in 1932, Hayden introduced the notion of metric connection with torsion in a Riemannian manifold [6]. Moreover, Yano studied semi-symmetric metric connection in a Riemannian manifold and obtained a result about conformally flatness [14].

The notion of semi symmetric connection was generalized to quarter symmetric connection by Golab in 1975 [5]. There are many papers about quarter symmetric connection not only in Riemannian manifolds but also in Hermitian, Kähler, Kenmotsu manifolds (see Mishra and Pandey [9], Dwivedi [3], Pusic [12], Yano and Imai [15]).

In this work, we consider a quarter symmetric connection on Kähler Weyl manifolds and almost contact Weyl manifolds and examine the properties of this connection.

\section{2}

PRELIMINARIES

A Weyl manifold is a differentiable manifold $M$ of dimension $n$ with a conformal metric tensor $g$ and a symmetric connection $D$ which satisfies, called the 
compatibility condition,

$$
D_{k} g_{i j}=2 \omega_{k} g_{i j} \text {, }
$$

where $\omega$ is a 1-form. Such a Weyl manifold is denoted by $M_{n}(g, \omega)$. If $\omega$ is a closed form, $M_{n}(g, \omega)$ is conformal to a Riemannian manifold.

Under the conformal change of the metric tensor $g$,

$$
\breve{g}_{i j}=\lambda^{2} g_{i j}, \quad \lambda>0,
$$

the 1 -form $\omega$ changes by the law

$$
\breve{\omega}_{k}=\omega_{k}+D_{k} \ln \lambda .
$$

A quantity $S$ is called a satellite of $g$ with weight $r$ if it admits a transformation of the form

$$
\breve{S}=\lambda^{r} S,
$$

under the change (2.2) of the metric tensor $g$.

The prolonged (extended) covariant derivative of a satellite $S$ of weight $r$ is defined by

$$
\dot{D}_{k} S=D_{k} S-r w_{k} S,
$$

from which it follows that $\dot{D}_{k} g_{i j}=0$ (see [16], [7], [10]).

It is easy to see from (2.1) that

$$
\Gamma_{k l}^{i}=\left\{\begin{array}{c}
i \\
k l
\end{array}\right\}-g^{i m}\left(g_{m k} \omega_{l}+g_{m l} \omega_{k}-g_{k l} \omega_{m}\right)
$$

where $\Gamma_{j k}^{i}$ are the coefficients of the Weyl connection $D$ and $\left\{\begin{array}{c}i \\ k l\end{array}\right\}$ are the connection coefficients of the Levi-Civita connection.

The mixed curvature tensor, the covariant curvature tensor, the Ricci tensor and the scalar curvature for $M_{n}(g, \omega)$ are respectively given by [11]:

$$
\begin{aligned}
v^{j} W_{j k l}^{p} & =\left(D_{k} D_{l}-D_{l} D_{k}\right) v^{p}, \\
W_{h j k l} & =g_{h p} W_{j k l}^{p}, \\
W_{i j} & =W_{i j p}^{p}=g^{h k} W_{h i j k}, \\
s & =g^{i j} W_{i j} .
\end{aligned}
$$

By considering (2.7), the explicit form of the mixed curvature tensor $W_{j k l}^{p}$ for $M_{n}(g, \omega)$ is

$$
W_{j k l}^{p}=\partial_{k} \Gamma_{j l}^{p}-\partial_{l} \Gamma_{j k}^{p}+\Gamma_{h k}^{p} \Gamma_{j l}^{h}-\Gamma_{h l}^{p} \Gamma_{j k}^{h} .
$$

The mixed curvature tensor, the covariant curvature tensor and the Ricci tensor of $M_{n}(g, \omega)$ satisfy the following properties [11]:

$$
\begin{aligned}
& W_{i j k l}+W_{i j l k}=0, \quad W_{i k l}^{i}=n\left(D_{l} \omega_{k}-D_{k} \omega_{l}\right)=2 n D_{[l} \omega_{k]}, \\
& W_{i j k l}+W_{j i k l}=4 g_{i j} D_{[l} \omega_{k]}, \quad W_{[i j]}=n D_{[i} \omega_{j]} .
\end{aligned}
$$


Let $N$ be an $n$-dimensional Riemannian manifold endowed with a linear connection $\nabla$. Then $\nabla$ is said to be quarter symmetric if the torsion tensor $T_{j k}^{i}$ of $\nabla$ satisfies

$$
T_{j k}^{i}=p_{j} A_{k}^{i}-p_{k} A_{j}^{i},
$$

where $p_{k}$ is a 1 -form and $A_{i}^{j}$ is any $(1,1)$ tensor field. If $A_{j k}$ is defined as $A_{j}^{i} g_{j k}=A_{j k}$, then

$$
A_{j k}=U_{j k}+V_{j k},
$$

where $U_{j k}$ and $V_{j k}$ are respectively symmetric and anti symmetric part of $A_{j k}$ [15].

\section{$3 \quad$ KäHLER WEYL MANIFOLDS}

A Weyl manifold of dimension $2 n$ is called Kähler Weyl if

$$
\begin{gathered}
F_{j}^{i} F_{i}^{h}=-\delta_{j}^{h}, \\
F_{j}^{t} F_{i}^{s} g_{t s}=g_{j i},
\end{gathered}
$$

and

$$
\dot{D}_{j} F_{i}^{k}=0, \quad \forall i, j, k,
$$

where $F_{i}^{j}$ is a $(1,1)$ tensor field of weight zero and called an almost complex structure, $g_{i j}$ is Hermitian metric. Such a manifold will be denoted by $K M_{2 n}(g, \omega)[2]$.

The $(0,2)$ tensor field $F_{i j}$ of weight 2 and $(2,0)$ tensor field $F^{i j}$ of weight -2 are, respectively, given by

$$
F_{i j}=F_{i}^{k} g_{k j}=-F_{j i}
$$

and

$$
F^{i j}=F_{k}^{j} g^{i k}=-F^{j i} .
$$

The contraction of (3.4) on the indices $k$ and $i$ gives $F_{i}^{i}=0$.

Suppose that a Kähler Weyl manifold admits a quarter symmetric linear connection $\bar{D}$ with the torsion tensor $\bar{T}$ and satisfies the following compatibility condition

$$
\bar{D}_{k} g_{i j}=2 \omega_{k} g_{i j} \text {. }
$$

If we take $U_{j k}=g_{j k}$ and $V_{j k}=F_{j k}$ in (2.15), then we find that $A_{i}^{k}=\delta_{i}^{k}+F_{i}^{k}$. Therefore, the torsion tensor $\bar{T}$ takes the form

$$
\bar{T}_{j k}^{i}=p_{j}\left(\delta_{k}^{i}+F_{k}^{i}\right)-p_{k}\left(\delta_{j}^{i}+F_{j}^{i}\right) .
$$

We note that the 1 -form $p_{k}$ is of zero weight.

Theorem 3.1. On every Kähler Weyl manifold there exists a unique quarter symmetric linear connection associated to every 1-form $p$ and $(1,1)$ tensor field F. 
Proof. Assume that the relation between $\bar{\Gamma}_{m k}^{j}$ and $\Gamma_{m k}^{j}$ is given by

$$
\bar{\Gamma}_{m k}^{j}=\Gamma_{m k}^{j}+U_{m k}^{j},
$$

where $\bar{\Gamma}_{m k}^{j}$ and $\Gamma_{m k}^{j}$ are respectively the coefficients of $\bar{D}$ and $D$, and $U_{m k}^{j}$ is any $(1,2)$ tensor field. Then from $(2.1),(3.6)$ and $(3.8)$, we find that

$$
U_{i k}^{h} g_{h j}+U_{j k}^{h} g_{h i}=0 .
$$

After permuting the indices $i, j$ and $k$ in the above equation cyclicly and using some algebraic operations, we obtain

$$
\begin{aligned}
\left(U_{i k}^{h}+U_{k i}^{h}\right) g_{h j} & =\left(U_{i j}^{h}-U_{j i}^{h}\right) g_{h k}+\left(U_{k j}^{h}-U_{j k}^{h}\right) g_{h i} \\
& =\bar{T}_{i j}^{h} g_{h k}+\bar{T}_{k j}^{h} g_{h i} .
\end{aligned}
$$

From (3.7) and after some simplifications, we have

$$
U_{i k}^{t}+U_{k i}^{t}=p_{i}\left(\delta_{k}^{t}-F_{k}^{t}\right)+p_{k}\left(\delta_{i}^{t}-F_{i}^{t}\right)-2 p^{t} g_{i k},
$$

where $p^{t}=p_{k} g^{t k}$. Since $\bar{T}_{i k}^{t}=U_{i k}^{t}-U_{k i}^{t}$, by considering the above equation, we obtain

$$
U_{i k}^{t}=p_{i} \delta_{k}^{t}-p_{k} F_{i}^{t}-p^{t} g_{i k} .
$$

Hence, we find that $\bar{\Gamma}_{m k}^{j}=\Gamma_{m k}^{j}+p_{m} \delta_{k}^{j}-p_{k} F_{m}^{j}-p^{j} g_{m k}$ which completes the proof.

The mixed curvature tensor $\bar{W}_{j k l}^{i}$ for $\bar{D}$ is given by

$$
\bar{W}_{j k l}^{i}=\partial_{k} \bar{\Gamma}_{j l}^{i}-\partial_{l} \bar{\Gamma}_{j k}^{i}+\bar{\Gamma}_{j l}^{m} \bar{\Gamma}_{m k}^{i}-\bar{\Gamma}_{j k}^{m} \bar{\Gamma}_{m k}^{i} .
$$

Hence, by considering the definition of $\bar{\Gamma}_{j l}^{i}$ and after a long straightforward calculations, we obtain

$$
\begin{aligned}
\bar{W}_{j k l}^{i} & =W_{j k l}^{i}+\delta_{l}^{i} \alpha_{j k}-\delta_{k}^{i} \alpha_{j l}+g_{j k} g^{i t} \alpha_{t l}-g_{j l} g^{i t} \alpha_{t k}-2 F_{j}^{i} \dot{D}_{[k} p_{l]} \\
& +p^{i}\left(F_{j k} p_{l}-F_{j l} p_{k}\right)+p_{j}\left(F_{k}^{i} p_{l}-F_{l}^{i} p_{k}\right),
\end{aligned}
$$

where $\alpha_{j k}=\dot{D}_{k} p_{j}-p_{j} p_{k}+F_{j}^{m} p_{m} p_{k}+\frac{1}{2} g_{j k} p^{m} p_{m}$.

Therefore, the covariant curvature tensor $\bar{W}_{i j k l}$, the Ricci tensor $\bar{W}_{j k}$ and the scalar curvature $\bar{s}$ are respectively given by

$$
\begin{aligned}
\bar{W}_{i j k l} & =W_{i j k l}+g_{i l} \alpha_{j k}-g_{i k} \alpha_{j l}+g_{j k} \alpha_{i l}-g_{j l} \alpha_{i k}+2 F_{i j} \dot{D}_{[k} p_{l]} \\
& +p_{i}\left(F_{j k} p_{l}-F_{j l} p_{k}\right)+p_{j}\left(F_{i l} p_{k}-F_{i k} p_{l}\right) \\
\bar{W}_{j k} & =W_{j k}+(n-2) \alpha_{j k}+g_{j k} g^{i l} \alpha_{i l}-g_{j l} \alpha_{i k}+2 g^{i l} F_{i j} \dot{D}_{[k} p_{l]} \\
& +F_{j k} p_{l} p^{l}-F_{j l} p^{l} p_{k}-F_{l k} p^{l} p_{j}
\end{aligned}
$$

and

$$
\bar{s}=s+2(n-1) g^{j k} \alpha_{j k}+2 F^{l k} \dot{D}_{[k} p_{l]} .
$$


Proposition 3.2. The mixed curvature tensor $\bar{W}_{i k l}^{i}$ and the covariant curvature tensor $\bar{W}_{i j k l}$ of a Kähler Weyl manifold with a quarter symmetric linear connection satisfy the following relations:

(i) $\bar{W}_{i j k l}+\bar{W}_{i j l k}=0$

(ii) $\bar{W}_{i j k l}+\bar{W}_{j i k l}=4 g_{i j} D_{[l} \omega_{k]}$

(iii) $\bar{W}_{i k l}^{i}=W_{i k l}^{i}=2 n D_{[l} \omega_{k]}$.

Proof. (i) The covariant curvature tensor $\bar{W}_{i j k l}$ of a Kähler Weyl manifold endowed with a quarter symmetric linear connection $\bar{D}$ is given by (3.15). By changing the indices $k$ and $l$ and then taking the sum of the equations obtained gives $\bar{W}_{i j k l}+\bar{W}_{i j l k}=W_{i j k l}+W_{i j l k}=0$.

(ii) Similarly, if we change the indices $k$ and $l$ in (3.15) and sum up the obtained equations, then we get $\bar{W}_{i j k l}+\bar{W}_{j i k l}=W_{i j k l}+W_{j i k l}=4 g_{i j} D_{[l} \omega_{k]}$.

(iii) Since $F_{i}^{i}=0$, the result follows easily from (3.14).

Theorem 3.3. If the curvature tensor of a Kähler Weyl manifold with a quarter symmetric linear connection vanishes and the 1-form $p_{k}$ is locally a gradient, then the connection reduces to the Weyl connection.

Proof. If $\bar{W}_{i j k l}=0$ and the 1 -form $p_{k}$ is locally a gradient, then (3.15) takes the form

$$
\begin{aligned}
W_{i j k l} & =-g_{i l} \alpha_{j k}+g_{i k} \alpha_{j l}-g_{j k} \alpha_{i l}+g_{j l} \alpha_{i k} \\
& -p_{i}\left(F_{j k} p_{l}-F_{j l} p_{k}\right)-p_{j}\left(F_{i l} p_{k}-F_{i k} p_{l}\right)
\end{aligned}
$$

If we permute the indices $j, k$ and $l$ in (3.18) cyclicly, then we obtain two more equations. Now, by taking the sum of the three equations and taking into account of the $1^{\text {st }}$ Bianchi Identity for Weyl manifolds, we obtain

$$
0=g_{i l} \alpha_{[j k]}+g_{i j} \alpha_{[k l]}+g_{i k} \alpha_{[l j]}+F_{j k} p_{i} p_{l}+F_{k l} p_{i} p_{j}+F_{l j} p_{i} p_{k} .
$$

By contracting the above equation with $F^{j l} g^{i k}$, we get for $n \neq 2$

$$
F^{j l} \alpha_{[j l]}=-p^{k} p_{k} .
$$

Since $\alpha_{[j l]}=\frac{1}{2}\left(F_{j m} p^{m} p_{l}-F_{l m} p^{m} p_{j}\right)$,

$$
\begin{aligned}
-p^{k} p_{k} & =\frac{1}{2} F^{j l}\left(F_{j m} p^{m} p_{l}-F_{l m} p^{m} p_{j}\right) \\
& =\frac{1}{2}\left(\delta_{m}^{l} p^{m} p_{l}+\delta_{m}^{j} p^{m} p_{j}\right) \\
& =p^{k} p_{k},
\end{aligned}
$$

from which we find that $p_{k}=0$ for positive definite metric tensors belonging to the conformal class. Now since $p_{k}=0, \bar{\Gamma}_{m k}^{j}=\Gamma_{m k}^{j}+p_{m} \delta_{k}^{j}-p_{k} F_{m}^{j}-p^{j} g_{m k}$ takes the form $\bar{\Gamma}_{m k}^{j}=\Gamma_{m k}^{j}$ which completes the proof. 


\section{$4 \quad$ Almost Contact Weyl Manifolds}

Let $M_{2 n+1}$ be a differentiable manifold of dimension $2 n+1$. An almost contact structure $(\phi, \xi, \eta)$ on $M_{2 n+1}$ is a triple satisfying the following relations

$$
\begin{aligned}
\phi_{i}^{j} \phi_{j}^{k} & =-\delta_{i}^{k}+\eta_{i} \xi^{k}, \\
\eta_{i} \xi^{i} & =1 \\
\phi_{i}^{j} \xi^{i} & =0 \\
\eta_{i} \phi_{j}^{i} & =0
\end{aligned}
$$

where $\phi_{i}^{j}$ is a tensor field of type $(1,1), \xi^{i}$ is a vector field and $\eta_{i}$ is a 1 -form. Moreover, if there is given a Riemannian metric $g_{i j}$ such that

$$
\begin{aligned}
g_{i j} \phi_{t}^{i} \phi_{s}^{j} & =g_{t s}-\eta_{t} \eta_{s}, \\
g_{i j} \xi^{j} & =\eta_{i},
\end{aligned}
$$

then $(\phi, \xi, \eta, g)$ is called an almost contact metric structure on $M$. A differentiable manifold $M_{2 n+1}$ with almost contact metric structure $(\phi, \xi, \eta, g)$ is called almost contact metric manifold [1].

It is easy to see that the tensor $\phi_{i j}$, which is defined by $\phi_{i}^{k} g_{j k}=\phi_{i j}$, is anti symmetric and contraction of $\phi_{i}^{j}$ gives $\phi_{i}^{i}=0$.

It follows immediately from the equations (4.1), (4.2) and (4.5) that the $(1,1)$ tensor field $\phi_{i}^{j}$, the 1 -form $\eta_{i}$ and the vector field $\xi^{i}$ are weight of 0,1 and -1 , respectively.

Let $M_{2 n+1}(g, \omega)$ be a Weyl manifold with the connection $D$. Then $M_{2 n+1}(g, \omega)$ has an almost contact structure if the following conditions are satisfied in addition to the conditions (4.1)-(4.6) [8]:

$$
\dot{D}_{k} g_{i j}=0, \quad \dot{D}_{k} \phi_{i}^{j}=0, \quad \dot{D}_{k} \eta_{i}=0, \quad \dot{D}_{k} \xi^{i}=0 .
$$

Such a manifold is called almost contact Weyl manifold and will be denoted by $A C M_{2 n+1}(g, \omega)$.

Now, we consider the manifold $A C M_{2 n+1}(g, \omega)$ with a quarter symmetric linear connection $\widetilde{D}$ and the torsion tensor $\widetilde{T}$ is of the form

$$
\widetilde{T}_{j k}^{i}=q_{j} \phi_{k}^{i}-q_{k} \phi_{j}^{i}
$$

where the $(1,1)$ tensor field $A_{i}^{j}=\phi_{i}^{j}$ and the 1 -form $q_{j}=f \eta_{j}$, where $f$ is any function of weight -1 . Here, we also have

$$
\widetilde{D}_{k} g_{i j}=2 \omega_{k} g_{i j}
$$

Theorem 4.1. On every almost contact Weyl manifold there exists a unique quarter symmetric linear connection associated to every 1 -form $q$ and $(1,1)$ tensor field $\phi$. 
Proof. Suppose that the relation between $\widetilde{\Gamma}_{m k}^{j}$ and $\Gamma_{m k}^{j}$ be

$$
\widetilde{\Gamma}_{m k}^{j}=\Gamma_{m k}^{j}+U_{m k}^{j}
$$

where $\widetilde{\Gamma}_{m k}^{j}$ and $\Gamma_{m k}^{j}$ are the coefficients of the connections $\widetilde{D}$ and $D$, respectively and $U_{m k}^{j}$ is any tensor field of type $(1,2)$.

From (2.1), (4.9) and (4.10) we have

$$
U_{i k}^{h} g_{h j}+U_{j k}^{h} g_{h i}=0 .
$$

Permuting cyclicly the indices $i, j, k$ and after some modifications, we obtain

$$
\begin{aligned}
\left(U_{i k}^{h}+U_{k i}^{h}\right) g_{h j} & =\left(U_{i j}^{h}-U_{j i}^{h}\right) g_{h k}+\left(U_{k j}^{h}-U_{j k}^{h}\right) g_{h i} \\
& =\widetilde{T}_{i j}^{h} g_{h k}+\widetilde{T}_{k j}^{h} g_{h i}
\end{aligned}
$$

Using the definition of $\widetilde{T}_{i j}^{h}$ and after straightforward calculations, we find that

$$
\left(U_{i k}^{h}+U_{k i}^{h}\right) g_{h j}=-q_{i} \phi_{k j}-q_{k} \phi_{i j} .
$$

Multiplying the last equation by $g^{j t}$, we have

$$
U_{i k}^{t}+U_{k i}^{t}=-q_{i} \phi_{k}^{t}-q_{k} \phi_{i}^{t}
$$

We conclude from the above equation that

$$
\widetilde{T}_{i j}^{h}=U_{i k}^{t}-U_{k i}^{t}=q_{i} \phi_{k}^{t}-q_{k} \phi_{i}^{t} .
$$

Hence, we obtain

$$
U_{i k}^{t}=-q_{k} \phi_{i}^{t},
$$

and therefore

$$
\widetilde{\Gamma}_{m k}^{j}=\Gamma_{m k}^{j}-q_{k} \phi_{m}^{j} .
$$

The mixed curvature tensor for an almost contact Weyl manifold endowed with a quarter symmetric linear connection $\widetilde{D}$ is of the form

$$
\widetilde{W}_{j k l}^{i}=\partial_{k} \widetilde{\Gamma}_{j l}^{i}-\partial_{l} \widetilde{\Gamma}_{j k}^{i}+\widetilde{\Gamma}_{j l}^{m} \widetilde{\Gamma}_{m k}^{i}-\widetilde{\Gamma}_{j k}^{m} \widetilde{\Gamma}_{m k}^{i} .
$$

By using (4.17) and (4.18) we have

$$
\begin{aligned}
\widetilde{W}_{j k l}^{i} & =\partial_{k}\left(\Gamma_{j l}^{i}-q_{l} \phi_{j}^{i}\right)-\partial_{l}\left(\Gamma_{j k}^{i}-q_{k} \phi_{k}^{i}\right) \\
& +\left(\Gamma_{j l}^{m}-q_{l} \phi_{j}^{m}\right)\left(\Gamma_{m k}^{i}-q_{k} \phi_{m}^{i}\right)-\left(\Gamma_{j k}^{m}-q_{k} \phi_{j}^{m}\right)\left(\Gamma_{m l}^{i}-q_{l} \phi_{m}^{i}\right) \\
& =W_{j k l}^{i}-\partial_{k}\left(q_{l} \phi_{j}^{i}\right)+\partial_{l}\left(q_{k} \phi_{j}^{i}\right)-q_{k} \Gamma_{j l}^{m} \phi_{m}^{i}-q_{l} \Gamma_{m k}^{i} \phi_{j}^{m} \\
& +q_{l} q_{k} \phi_{j}^{m} \phi_{m}^{i}+q_{l} \Gamma_{j k}^{m} \phi_{m}^{i}+q_{k} \Gamma_{m l}^{i} \phi_{j}^{m}-q_{k} q_{l} \phi_{j}^{m} \phi_{m}^{i}
\end{aligned}
$$


After some simplifications we obtain

$$
\begin{aligned}
\widetilde{W}_{j k l}^{i} & =W_{j k l}^{i}+\phi_{j}^{i}\left(\partial_{l} q_{k}-\partial_{k} q_{l}\right)+q_{k}\left(\partial_{l} \phi_{j}^{i}-\Gamma_{j l}^{m} \phi_{m}^{i}+\Gamma_{m l}^{i} \phi_{j}^{m}\right) \\
& -q_{l}\left(\partial_{k} \phi_{j}^{i}-\Gamma_{j k}^{m} \phi_{m}^{i}+\Gamma_{m k}^{i} \phi_{j}^{m}\right) .
\end{aligned}
$$

If we use the definition of prolonged covariant derivative for $\phi_{j}^{i}$ and $\eta_{k}$, then we get

$$
\widetilde{W}_{j k l}^{i}=W_{j k l}^{i}+\phi_{j}^{i}\left(\dot{D}_{l} q_{k}-\dot{D}_{k} q_{l}\right)+q_{k} \dot{D}_{l} \phi_{j}^{i}-q_{l} \dot{D}_{k} \phi_{j}^{i}
$$

Since $\dot{D}_{l} \phi_{j}^{i}=0$, we find that

$$
\widetilde{W}_{j k l}^{i}=W_{j k l}^{i}+\phi_{j}^{i}\left(\dot{D}_{l} q_{k}-\dot{D}_{k} q_{l}\right) .
$$

From (4.22), the covariant curvature tensor $\widetilde{W}_{i j k l}$ is given by

$$
\widetilde{W}_{i j k l}=W_{i j k l}+2 \phi_{j i} \dot{D}_{[l} q_{k]},
$$

where $\dot{D}_{[l} q_{k]}$ is anti symmetric part of $\dot{D}_{l} q_{k}$.

Contracting the tensor $\widetilde{W}_{j k l}^{i}$ with respect to $i$ and $l$ and using the fact that $\eta_{i} \phi_{j}^{i}=0$, gives us

$$
\widetilde{W}_{j k}=W_{j k}+\phi_{j}^{i} \dot{D}_{i} q_{k}
$$

Theorem 4.2. For an almost contact Weyl manifold with a quarter symmetric linear connection, we have

$$
\widetilde{s}=s,
$$

where $\widetilde{s}$ and s are scalar curvature of the manifold with respect to the connections $\widetilde{D}$ and $D$, respectively.

Proof. Multiplying (4.24) by $g^{j k}$ and using the identity $\xi^{j} \phi_{j}^{i}=0$ gives

$$
\begin{aligned}
\widetilde{s} & =s+\dot{D}_{i}\left(q_{k} g^{j k} \phi_{j}^{i}\right) \\
& =s+\dot{D}_{i}\left(f \eta_{k} g^{j k} \phi_{j}^{i}\right) \\
& =s+\dot{D}_{i}\left(f \xi^{j} \phi_{j}^{i}\right) \\
& =s .
\end{aligned}
$$

Theorem 4.3. On an almost contact Weyl manifold with a quarter symmetric linear connection, if the 1-form $q$ is locally a gradient, then

$$
\begin{aligned}
\widetilde{W}_{j k l}^{i} & =W_{j k l}^{i}, \\
\widetilde{W}_{j k} & =W_{j k} .
\end{aligned}
$$

Proof. The proof is immediate from (4.22). 
Proposition 4.4. On an almost contact Weyl manifold with a quarter symmetric connection, the following relations hold:

(i) $\widetilde{W}_{i j k l}+\widetilde{W}_{i j l k}=0$

(ii) $\widetilde{W}_{i j k l}+\widetilde{W}_{j i k l}=\frac{4}{n} g_{i j} W_{[l k]}$

(iii) $\widetilde{W}_{i k l}^{i}=2 n D_{[l} \omega_{k]}$

(iv) $\widetilde{W}_{k l i j}+\widetilde{W}_{k i j l}+\widetilde{W}_{k j l i}=2\left(\phi_{k l} \dot{D}_{[i} q_{j]}+\phi_{k i} \dot{D}_{[j} q_{l]}+\phi_{k j} \dot{D}_{[l} q_{i]}\right)$

Proof. (i). Changing the indices $k$ and $l$ in (4.23) yields

$$
\widetilde{W}_{i j l k}=W_{i j l k}-2 \phi_{j i} \dot{\nabla}_{[l} q_{k]} .
$$

By adding (4.23) to (4.26) we obtain the result.

(ii). Similar to proof $(i)$.

(iii). Contracting (4.22) with respect to $i$ and $j$ yields

$$
\widetilde{W}_{i k l}^{i}=W_{i k l}^{i}+\phi_{i}^{i}\left(\dot{D}_{l} q_{k}-\dot{D}_{k} q_{l}\right) .
$$

Since $\phi_{i}^{i}=0$, we find that

$$
\widetilde{W}_{i k l}^{i}=W_{i k l}^{i}=2 n D_{[l} \omega_{k]} .
$$

(iv). Using the $1^{\text {st }}$ Bianchi Identity

$$
W_{k l i j}+W_{k i j l}+W_{k j l i}=0,
$$

and after the straightforward calculations, we get the result.

\section{REFERENCES}

[1] Blair, D. E., Riemannian Geometry of Contact and Symplectic Manifolds(second edition), Progress in Mathematics, Birkhauser, 2010.

[2] Demirbüker, H., Özdemir, F., Almost Hermitian, Almost Kaehlerian and Almost Semi-Kaehlerian Structures in Weyl spaces. Buletinnul Sthntific Univ. Politeh. Din Timisoara Math. 43 (1998), 1-7.

[3] Dwivedi, M. K., A quarter symmetric non-metric connection in a Kenmotsu manifold, Int. Electron. J. Geom. 4 (2011), 115-124.

[4] Friedmann, A., Schouten, J. A., Überdie geometric der holbsymmetricschen Übertragurgen, Math. Zeitschr. 21 (1924),211-239.

[5] Golab, S., On semi-symmetric and quarter symmetric linear connections, Tensor N.S. 29 (1975), 249-254. 
[6] Hayden, H. A., Subspace of space with torsion, Proc. Lond. Math. Soc. 34 (1932), 27-50.

[7] Hlavaty, V., Theorie d'immersion d'une $W_{m}$ dans $W_{n}$. Ann Soc Polon. Math 21 (1949), 196-206.

[8] Matzeu, P., Almost Contact Einstein-Weyl Structures, Manuscripta Math. 108 (2002), 275-288.

[9] Mishra, R. S., Pandey, S. N., On quarter symmetric metric F-connections, Tensor N.S. 34 (1980), 1-7.

[10] Norden, A., Affinely Connected Spaces, Nauka, Moscow, 1976.

[11] Özdeğer, A., On sectional curvatures of Weyl manifolds. Proc Japan Acad 82 (2006), 123-125.

[12] Pusic, N., On quarter-symmetric metric connections on a hyperbolic Kaehlerian space, Publ. Inst. Math. (Beograd) (N.S.), 73(87) (2003), 73-80.

[13] Weyl, H., Raum-Zeit-Materie, Springer, Berlin 1918.

[14] Yano, K., On semi-symmetric metric connection, Rev. Roum. Math. Pures. et Appl. 15 (1970), 1579-1586.

[15] Yano, K., Imai, T., Quarter-symmetric metric connections and their curvature tensors. Tensor, N.S. 38 (1982), 13-18.

[16] Zlatanov, G., Nets in the n-dimensional Space of Weyl. C.R. Acad. Bulg. Sci. 41 (1988), 29-32. 